\title{
THE MONOTONE CLASS THEOREM IN INFINITARY LOGIC 1
}

\author{
H. JEROME KEISLER ${ }^{2}$
}

\begin{abstract}
A monotone formula in the infinitary logic $L_{\omega, \omega}$ is a formula which is built up from finite formulas using only quantifiers and monotone countable conjunctions and disjunctions. The monotone class theorem from measure theory is used to show that every formula of $L_{\omega, \omega}$ is logically equivalent to a monotone formula (the monotone normal form theorem). The proof is effectivized in order to obtain similar normal form theorems for admissible logics $L_{A}$.
\end{abstract}

0 . Introduction. The monotone class theorem, that the $\sigma$-field generated by a field of sets $S$ coincides with the monotone class generated by $S$, is a familiar tool in measure theory. It may be interpreted as a normal form theorem for propositional logic with countable conjunctions and disjunctions: Every sentence is logically equivalent to a sentence built up from finite sentences using monotone conjunctions and disjunctions. The purpose of this note is to point out that there are similar normal form theorems for richer infinitary logics. This work grew out of our development of logic with probability quantifiers in [3]. Here we discuss more traditional infinitary logics. In $\$ 1$ we shall prove a monotone normal form theorem for the logic $L_{\omega_{1} \omega}$ which is obtained from first-order logic by adding countable conjunctions and disjunctions. In $\$ 2$ we prove a monotone normal form theorem for the sublogics $L_{A}$ of $L_{\omega_{1} \omega}$ where $A$ is a locally countable admissible set. For this purpose we need an "effective" proof of the monotone class theorem. In $\$ 3$ we obtain analogous normal form theorems for the infinitary logic $L_{\infty \infty \omega}$, which has arbitrary conjunctions and disjunctions, and for its admissible sublogics.

For background material on infinitary logic and admissible sets see Barwise [1]. We use the convention that $\varphi(\vec{x})$ denotes a formula $\varphi$ whose free variables form a subset of the $n$-tuple $\vec{x}=\left\langle x_{1}, \ldots, x_{n}\right\rangle$.

I wish to thank J. Barwise, E. Fisher, and K. Kunen for useful discussions of this work.

1. The logic $L_{\omega_{1} \omega}$. The monotone class theorem may be stated in terms of Boolean algebras as follows.

\subsection{Monotone Class Theorem. Let B be a countably complete Boolean}

Received by the editors December 13, 1976.

AMS (MOS) subject classifications (1970). Primary $02 \mathrm{H} 05$.

${ }^{1}$ This research was supported in part by National Science Foundation Grant GP-43882X.

2 The author is a John Simon Guggenheim Fellow. 
algebra and let $B_{0}$ be a Boolean subalgebra of $B$. Let $C\left(B_{0}\right)$ be the countably complete Boolean subalgebra of $B$ generated by $B_{0}$. Then $C\left(B_{0}\right)$ is equal to the intersection of all $M$ such that $B_{0} \subseteq M \subseteq B$ and $M$ is closed under suprema of countable increasing sequences and infima of countable decreasing sequences.

For a proof see Halmos [2]. An earlier reference is Saks [4, p. 85].

1.2. Definition. A countable conjunction $\wedge_{n<\omega} \varphi_{n}$ in $L$ is said to be monotone if for each $n<\omega$, $\vDash \varphi_{n+1} \rightarrow \varphi_{n}$. A countable disjunction $V_{n}<\omega \varphi_{n}$ is monotone if for each $n<\omega, \vDash \varphi_{n} \rightarrow \varphi_{n+1}$. The set of monotone formulas of $L_{\omega_{1} \omega}$ is the least set $M$ such that every finite formula belongs to $M, M$ is closed under quantifiers, and $M$ is closed under monotone conjunctions and disjunctions.

1.3. COROLlaRY. Every quantifier-free formula $\varphi(\vec{x})$ of $L_{\omega_{1} \omega}$ is logically equivalent to a monotone quantifier-free formula $\psi(\vec{x})$.

Proof. Let $\Phi(\vec{x})$ be the set of all quantifier-free formulas with at most the free variables $\vec{x}$. Identifying logically equivalent formulas we obtain a countably complete Boolean algebra $B$. The finite formulas of $\Phi(\vec{x})$ determine a Boolean subalgebra $B_{0}$ which countably generates $B$. The result now follows from the monotone class theorem.

1.4. Monotone Normal Form TheOrem FOR $\mathbf{L}_{\omega_{1} \omega_{\vec{x}}}$ Every formula $\varphi(\vec{x})$ of $L_{\omega_{1} \omega}$ is logically equivalent to a monotone formula $\psi(\vec{x})$.

Proof. Let $S_{0}$ be the set of finite formulas. We define sets of formulas $S_{\alpha}, \alpha$ an ordinal, as follows. For each $\alpha$,

$T_{\alpha}=$ closure of $S_{\alpha}$ under finite connectives.

$U_{\alpha}=$ closure of $T_{\alpha}$ under countable $\wedge, \vee, \neg$.

$S_{\alpha+1}=$ closure of $U_{\alpha}$ under quantifiers.

For limit ordinals $\lambda, S_{\lambda}=\cup_{\beta<\lambda} S_{\beta}$. Given an $n$-tuple of variables $\vec{x}$, let $S_{\alpha}(\vec{x})$ be the set of $\varphi(\vec{x}) \in S_{\alpha}$ with at most $\vec{x}$ free, and define $T_{\alpha}(\vec{x}), U_{\alpha}(\vec{x})$ similarly. We show by induction on $\alpha$ that each $\varphi(\vec{x}) \in U_{\alpha}(\vec{x})$ is logically equivalent to a monotone formula in $U_{\alpha(\vec{x})}$. (For $\alpha=0$ this is Corollary 1.3.) Assume the result for all $\beta<\alpha$ and all $\vec{x}$. Let $U=U_{\beta}$ if $\alpha=\beta+1$, and $U$ $=S_{\alpha}$ if $\alpha$ is a limit ordinal. $S_{\alpha}$ is the closure of $U$ under quantifiers, and $U$ is closed under $\wedge, \vee, \neg$, and free substitution. Using the proof of the prenex normal form theorem, we see that every $\varphi(\vec{x}) \in T_{\alpha}(\vec{x})$ is logically equivalent to some $\psi(\vec{x}) \in S_{\alpha}(\vec{x})$. Therefore, identifying logically equivalent formulas, $S_{\alpha}(\vec{x})$ is a Boolean subalgebra of the countably complete Boolean algebra $U_{\alpha}(\vec{x})$, and $S_{\alpha}(\vec{x})$ countably generates $U_{\alpha}(\vec{x})$. By the monotone class theorem, each $\varphi(\vec{x}) \in U_{\alpha}(\vec{x})$ is logically equivalent to a formula $\psi(\vec{x})$ built up from formulas of $S_{\alpha}(\vec{x})$ using only monotone conjunctions and disjunctions. By the inductive hypothesis, when $\alpha>0, \psi(\vec{x})$ is equivalent to a formula $\theta(\vec{x})$ built up from monotone formulas of $\cup_{\beta<\alpha} U_{\beta}(\vec{x})$ using only quantifiers and monotone conjunctions and disjunctions. Then $\theta(\vec{x})$ is a monotone formula of $U_{\alpha}(\vec{x})$, and our induction is complete. Since each formula of $\mathbf{L}_{\omega_{1} \omega}$ 
belongs to some $U_{\alpha}(\vec{x})$, the result follows.

2. Locally countable admissible logics. Consider an admissible set $A$ $=\langle M, A, \in, \ldots\rangle$ over a model $M$ in the sense of Barwise [1]. $A$ is said to be locally countable if $\omega \in A$ and for each $a \in A$, either $a$ is finite or there is an $f \in A$ such that $f$ is a bijection of $\omega$ onto $a$. $A$ is said to be recursively listed if there is an $A$-recursive bijection of $o(A)$ onto $M \cup A$. Here $o(A)$ is the least ordinal not in $A$. The admissible logic $L_{A}$ is defined as the set of all formulas of $L_{\infty \omega}$ which belong to $A$. If $A$ is locally countable, $L_{A}$ is included in $L_{\omega_{1} \omega}$.

2.1. TheOrem. Let $A$ be a locally countable admissible set .

(i) Every formula $\varphi \overrightarrow{(x)} \in L_{A}$ is logically equivalent to a monotone formula $\psi \overrightarrow{(x)} \in L_{A}$.

(ii) If $A$ is recursively listed, then there is an A-recursive function $F: L_{A} \rightarrow L_{A}$ such that for each $\varphi(\vec{x}) \in L_{A}, F \varphi$ is a monotone formula $\psi(\vec{x}) \in L_{A}$ which is logically equivalent to $\varphi(\vec{x})$.

Proof. Let us call a formula normal if it belongs to the least set of formulas which contains the finite formulas and is closed under quantifiers, finite conjunctions and disjunctions, and monotone conjunctions and disjunctions. Using the fact that $A$ is locally countable, one can prove that every $\varphi(\vec{x}) \in L_{A}$ is logically equivalent to a normal formula in $L_{A}$. The idea is to first move all negation symbols inside and then, starting from the inside, replace infinite conjunctions $\wedge_{n<\omega} \varphi_{n}$ by the equivalent monotone conjunctions $\wedge_{n<\omega}\left(\wedge_{m \leqslant n} \varphi_{m}\right)$, and similarly for disjunctions. Let $K_{\infty \omega}$ be the least class of formulas of $L_{\infty \omega}$ which contains the finite formulas and is closed under quantifiers, and finite and infinite conjunctions and disjunctions. Obviously, every normal formula belongs to $K_{\infty \omega}$. We define a mapping $G: K_{\infty \omega} \rightarrow K_{\infty \omega}$ with the following properties :

(1) For every $\varphi(\vec{x}) \in K_{\infty \omega}, G \varphi$ is a formula $\psi(\vec{x})$ which is logically equivalent to $\varphi(\vec{x})$.

(2) If $\varphi(\vec{x})$ is normal, then $G \varphi$ is monotone.

(3) The restriction of $G$ to $A$ is an $A$-recursive function.

We define $G$ as follows.

If $\varphi$ is finite, $G \varphi=\varphi$.

$G(\forall x \varphi)=\forall x G(\varphi)$, and similarly for $\exists$, where $\varphi$ is infinite.

$G\left(\wedge_{s \in S} \varphi_{s}\right)=\wedge_{s \in S} G\left(\varphi_{s}\right)$, and similarly for $\vee$.

When $\varphi$ is infinite and $\psi$ is finite, $G(\varphi \wedge \psi)$ is defined by:

$G\left(\left(\forall x \varphi_{0}\right) \wedge \psi\right)=\forall x G\left(\varphi_{0} \wedge \psi\right), x$ not free in $\psi$, and similarly for $\exists$.

$G\left(\left(\wedge_{s \in S} \varphi_{s}\right) \wedge \psi\right)=\wedge_{s \in S} G\left(\varphi_{s} \wedge \psi\right)$, and similarly for $\vee$.

$G\left(\left(\varphi_{1} \wedge \varphi_{2}\right) \wedge \psi\right)=G\left(G\left(\varphi_{1} \wedge \varphi_{2}\right) \wedge \psi\right)$, and similarly for $\varphi_{1} \vee \varphi_{2}$.

When $\psi$ is infinite, $G(\varphi \wedge \psi)$ is defined by:

$G\left(\varphi \wedge\left(\forall x \psi_{0}\right)\right)=\forall x G\left(\varphi \wedge \psi_{0}\right), x$ not free in $\varphi$, and similarly for $\exists$.

$G\left(\varphi \wedge\left(\wedge_{s \in S} \psi_{s}\right)\right)=\wedge_{s \in S} G\left(\varphi \wedge \psi_{s}\right)$, and similarly for $\vee$

$G\left(\varphi \wedge\left(\psi_{1} \wedge \psi_{2}\right)\right)=G\left(\varphi \wedge G\left(\psi_{1} \wedge \psi_{2}\right)\right)$, and similarly for $\psi_{1} \vee \psi_{2}$.

Finally, $G(\varphi \vee \psi)$ is defined in a dual manner. 
To show that the mapping $G$ is well defined, we introduce the rank function $r$ from $K_{\infty \omega}$ to the ordinals by

$$
\begin{aligned}
r(\varphi) & =0 \quad \text { if } \varphi \text { is finite, } \\
r(\varphi \wedge \psi) & =r(\varphi \vee \psi)=\omega(r(\varphi)+r(\psi)), \\
r(\forall x \varphi) & =r(\exists x \varphi)=r(\varphi)+1 \quad \text { if } \varphi \text { if infinite, } \\
r\left(\hat{S}_{s \in S} \varphi_{S}\right) & =r\left(\bigvee_{s \in S} \varphi_{S}\right)=\sup _{s \in S} r\left(\varphi_{s}\right)+1 .
\end{aligned}
$$

It can be shown by induction on the rank of formulas that the mapping $G$ up to a given rank exists uniquely, that $r(G(\varphi)) \leqslant r(\varphi)$, and that whenever a conjunction $\varphi \wedge \psi$ is an infinite formula,

$$
r(G(\varphi \wedge \psi))<r(\varphi \wedge \psi)
$$

We observe that $G(\forall x \varphi)$ and $G\left(\wedge_{s \in S} \varphi_{s}\right)$ are defined in terms of $G$ applied to formulas of smaller rank. The proof of (4) depends on the fact that if $\psi$ is finite then the rank of $\varphi \wedge \psi$ increases with the rank of $\varphi$, while in general the rank of $\varphi \wedge \psi$ increases with the rank of $\psi$. A routine induction on the rank $r(\varphi)$ shows that $G$ has properties (1) and (2). To prove (3), that the restriction of $G$ to $A$ is an $A$-recursive function, we use Gandy's Theorem on inductive definitions (see [1, p. 208]). It suffices to show that the restriction of $G$ to $A$ is the least fixed point of an $R$-positive $\Sigma$ formula $\theta(R, u, v) . \theta(R, u, v)$ is a finite disjunction with one disjunct for each clause in the definition of $G$. For example, the clause corresponding to

$$
G\left(\varphi \wedge\left(\psi_{1} \wedge \psi_{2}\right)\right)=G\left(\varphi \wedge G\left(\psi_{1} \wedge \psi_{2}\right)\right)
$$

is

$\exists u_{1} \exists w_{1} \exists w_{2} \exists v_{1}\left[u=u_{1} \wedge\left(w_{1} \wedge w_{2}\right)\right.$ and $R\left(w_{1} \wedge w_{2}, v_{1}\right)$ and $\left.R\left(u_{1} \wedge v_{1}, v\right)\right]$.

To prove part (i) of the theorem, let $\varphi(\vec{x}) \in L_{A}$ and choose a normal formula $\psi(\vec{x}) \in L_{A}$ logically equivalent to $\varphi(\vec{x})$. Then $G \psi$ is a monotone formula in $L_{A}$ which is logically equivalent to $\varphi(\vec{x})$. To prove (ii) we use the fact that $A$ is recursively listed to find an $A$-recursive function $H$ with the following property. For each $\varphi(\vec{x}) \in L_{A}, H \varphi$ is a normal formula $\psi(\vec{x})$ in $L_{A}$ which is logically equivalent to $\varphi(\vec{x})$. Then the $A$-recursive function $F(\varphi)$ $=G(H(\varphi))$ has the required property.

The above proof, when restricted to infinitary propositional logic, gives an effective proof of the monotone class theorem in the sense of admissible sets.

3. General admissible logics. By changing our notion of monotone, we can adapt the proof in the preceding section to admissible sets which are not locally countable. We must deal with formulas of $L_{\infty \omega}$ instead of $L_{\omega_{1} \omega}$. We consider two generalizations of the notion of a monotone formula. 
3.1. Definition. A conjunction $\wedge \Phi$ in $L_{\infty}$ is said to be weakly monotone if there is an enumeration $\Phi=\left\{\varphi_{\beta}: \beta<\alpha\right\}$ for some ordinal $\alpha$ such that whenever $\gamma<\beta<\alpha, \vDash \varphi_{\beta} \rightarrow \varphi_{\gamma}$. Weakly monotone disjunctions are defined dually. A weakly monotone formula is a formula of $L_{\infty}$ which is built up from finite formulas using quantifiers and weakly monotone conjunctions and disjunctions.

3.2. THEOREM. Every formula $\varphi(\vec{x})$ of $L_{\infty \omega}$ is logically equivalent to a weakly monotone formula $\psi(\vec{x})$ of $L_{\infty \omega}$.

An admissible set $A$ is said to be locally well-orderable if for every $a \in A$ there is an ordinal $\beta \in A$ and an $f \in A$ such that $f$ is a bijection of $\beta$ onto $a$.

3.3. THeorem. Suppose $A$ is a locally well-orderable admissible set.

(i) Every formula $\varphi(\vec{x}) \in L_{A}$ is logically equivalent to a weakly monotone formula $\psi(\vec{x}) \in L_{A}$.

(ii) If $A$ is recursively listed, then there is an A-recursive function $F: L_{A} \rightarrow L_{A}$ such that for each $\varphi(\vec{x}) \in L_{A}, F \varphi$ is a weakly monotone formula $\psi(\vec{x})$ which is logically equivalent to $\varphi(\vec{x})$.

Our second generalization was suggested by K. Kunen. It leads to a normal form theorem for arbitrary admissible logics.

3.4. Definition. A conjunction $\wedge \Phi$ in $L_{\infty \infty \omega}$ is said to be directed if for all $\varphi_{1}, \varphi_{2} \in \Phi$ there exists $\varphi \in \Phi$ such that $\vDash \varphi \rightarrow\left(\varphi_{1} \wedge \varphi_{2}\right)$. Directed disjunctions are defined in a dual manner, with $\vDash\left(\varphi_{1} \vee \varphi_{2}\right) \rightarrow \varphi$. A directed formula is a formula of $L_{\infty \omega}$ which is built up from finite formulas using quantifiers and directed conjunctions and disjunctions.

3.5. THEOREM. Let $A$ be an admissible set. There is an A-recursive function $F: L_{A} \rightarrow L_{A}$ such that for each $\varphi(\vec{x}) \in L_{A}, F \varphi$ is a directed formula $\psi(\vec{x})$ which is logically equivalent to $\varphi(\vec{x})$.

The proofs of Theorems 3.2, 3.3, and 3.5 all use the $A$-recursive function $G$ introduced in the proof of Theorem 2.1. In each case we modify the proof by changing the definition of a normal formula in the natural way. Theorem 3.5 depends on the fact that the process of replacing an infinite conjunction $\wedge \Phi$ in $A$ by the directed conjunction $\wedge\left\{\wedge \Phi_{0}: \Phi_{0}\right.$ is a finite subset of $\left.\Phi\right\}$ is $A$ recursive.

All the results in this paper still hold for infinitary logics with additional quantifiers $(Q x)$ provided that

$$
\begin{gathered}
\vDash(Q x) \varphi(x, \vec{z}) \leftrightarrow(Q y) \varphi(y, \vec{z}), \\
\vDash((Q x) \varphi(x)) \wedge \psi \leftrightarrow(Q x)(\varphi(x) \wedge \psi), \\
\vDash((Q x) \varphi(x)) \vee \psi \leftrightarrow(Q x)(\varphi(x) \vee \psi) .
\end{gathered}
$$

Monotone formulas should then be defined so as to be closed under both $(Q x)$ and $\neg(Q x) \neg$. The results also carry over to logics with quantifiers over 
sets of variables, including $L_{\omega_{1} \omega_{1}}$ and $L_{\infty \infty}$.

\section{REFERENCES}

1. J. Barwise, Admissible sets and structures, Springer-Verlag, Berlin and New York, 1975.

2. P. Halmos, Measure theory, Van Nostrand, Princeton, N. J., 1950. MR 11, 504.

3. H. J. Keisler, Hyperfinite model theory, (Proc. 1976 Oxford Logic Sympos.), (to appear).

4. S. Saks, Theory of the integral, Warsaw, 1937.

Department of Mathematics, University of Wisconsin, MAdison, Wisconsin 53706 